\title{
Physics Prospects at SuperKEKB/Belle II
}

\author{
Alessandro Gaz* \\ KMI, Nagoya University \\ E-mail: gaz@hepl.phys.nagoya-u.ac.jp
}

The Belle II experiment is in its final phase of construction and the SuperKEKB accelerator has successfully completed the first phase of commissioning. With an expected integrated luminosity of $50 \mathrm{ab}^{-1}$ to be collected by the year 2025, the Belle II dataset will allow us to probe New Physics scales that are well beyond the reach of direct production at the LHC and will complement the searches through indirect effects that are currently ongoing or planned. An overview of the golden channels, of their physics motivations, and of the expected sensitivity of the Belle II experiment will be given, with particular emphasis on the dataset that will be available in first two years of data taking.

The 3rd International Symposium on "Quest for the Origin of Particles and the Universe" 5-7 January 2017

Nagoya University, Japan

${ }^{*}$ Speaker. 


\section{Introduction}

The Physics prospects of the Belle II experiment at the SuperKEKB high luminosity $e^{+} e^{-}$ collider are presented here. After briefly summarizing in Section 2 the achievements of the first generation of $B$-Factories, the SuperKEKB accelerator and the Belle II detector will be described in Sections 3 and 4, respectively. Some Physics topics in which Belle II is expected to lead the progress in the next few years will be presented in Section 5, and the plans for the commissioning will be illustrated in Section 6 .

\section{The heritage from the $B$-Factories}

The BaBar and Belle experiments operated for about one decade, starting in the year 1999, at the asymmetric energy $e^{+} e^{-}$colliders PEP-II at the SLAC National Accelerator Laboratory of Menlo Park (USA) and KEKB at the High Energy Accelerator Research Organization in Tsukuba (Japan). They operated at a center of mass energy corresponding (or close to) the mass of the $\Upsilon(4 S)$ resonance, collecting in total an integrated luminosity of $0.5 \mathrm{ab}^{-1}$ (BaBar) and $1.0 \mathrm{ab}^{-1}$ (Belle).

Many important physics results have been achieved by the two Collaborations [1], they discovered $C P$-violation phenomena in the $B$-meson system, observed for the first time the $D^{0}-\bar{D}^{0}$ oscillation, discovered new exotic particles, and overall put very stringent constraints on possible New Physics scenarios by performing a broad array of measurements on (rare) decays of $B, D$, and $\tau$ particles. The CKM paradigm has been validated in a spectacular fashion, proving that the non trivial phase in the CKM Matrix is the source of all the $C P$-violation phenomena that have been observed so far in Particle Physics and this led to M. Kobayashi and T. Maskawa being awarded the Nobel Prize in the year 2008.

Although no significant evidence of New Physics was found at the $B$-Factories, some tensions with respect to the Standard Model predictions have been found in some channels. These will be thoroughly investigated by the upcoming Belle II experiment, as will be discussed in Section 5 .

\section{The SuperKEKB $e^{+} e^{-}$Collider}

The critical aspect for the success of the Belle II Physics Program relies on increasing the integrated luminosity to be collected by about a factor of 50, compared to what has been recorded by Belle. The KEKB accelerator has been upgraded to SuperKEKB, with the goal of achieving an instantaneous luminosity of $8 \times 10^{35} \mathrm{~cm}^{-2} \mathrm{~s}^{-1}$ (compared to its previous luminosity of $2.1 \times 10^{34}$ $\mathrm{cm}^{-2} \mathrm{~s}^{-1}$ ) and delivering an integrated luminosity of $50 \mathrm{ab}^{-1}$ by the year 2025, see Fig. 1.

Most of the expected improvement in the performance of SuperKEKB [2] will derive from the adoption of the nano-beam scheme [3]. The transverse size of the colliding bunches will be reduced by a factor $\sim 20$ compared to KEKB, and the beam currents will be increased by a factor 2 , for an overall gain in instantaneous luminosity of a factor $\sim 40$.

The construction of the SuperKEKB accelerator ring was completed in 2015 and the first circulation of beams was achieved in February 2016. Until July 2016, the machine has been operated with increasing currents, with the aim of gaining experience with the new equipment, improving the quality of the vacuum in the beam pipes, and studying the related backgrounds with BEAST II commissioning detector, an apparatus specifically designed for this purpose. 


\section{SuperKEKB luminosity projection}

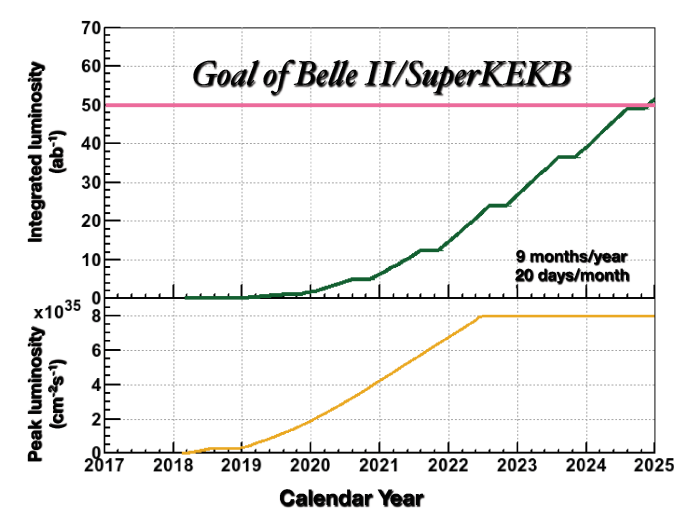

Figure 1: Projection for the integrated (top) and instantaneous (bottom) luminosity profile for the SuperKEKB project.

\section{The Belle II Detector}

The Belle II detector is an extensive upgrade (see Fig. 2) of the previous Belle detector (of which only the iron yoke, the solenoid, and the crystals of the electromagnetic calorimeter will be re-used). While the detailed description can be found in [4], here we will focus only on some of the most distinctive features of the new apparatus.

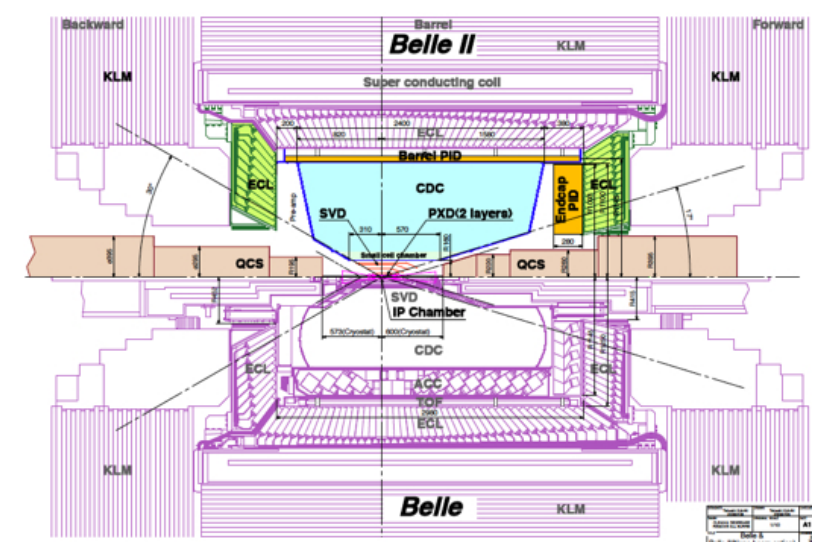

Figure 2: Layout of the Belle (bottom part of the figure) and Belle II (top) detectors.

The inner tracking device is constituted by two layers of DEPFET silicon pixel sensors (PXD), and four layers of silicon strip sensors (SVD). The innermost PXD layer will be positioned at a 14 $\mathrm{mm}$ distance from the interaction point (compared to $20 \mathrm{~mm}$ of Belle), allowing for a sizable improvement of the vertex resolution, while the outer layer of the SVD will be placed at a distance of $135 \mathrm{~mm}$, so that also the $K_{S}^{0}$ reconstruction efficiency and vertexing quality will be increased.

Particle identification (PID) in the barrel region will rely on the Time Of Propagation (TOP) counter, which determines the aperture angle of the Cherenkov photons, emitted by the charged 
particles while traversing its $2 \mathrm{~cm}$ thick quartz bars, by precisely measuring their time of arrival. Compared to the old ACC and TOF counters of Belle, the material budget is strongly reduced and the $K / \pi$ separation capabilities will improve from the typical $90 \%$ (10\%) of efficiency (fake rate) to $95 \%(5 \%)$.

\section{Selected Physics Topics}

In this section, a limited selection of Physics topics particularly relevant in the Belle II Physics Program will be covered. Emphasis will be given to the areas where the clean environment given by the $e^{+} e^{-}$collisions will be particularly advantageous over the larger statistics that can be obtained at the $\mathrm{LHCb}$ experiment.

\subsection{Time dependent $C P$-Violation}

Time-dependent $C P$-violation phenomena were one of the strongest motivations for the construction of the first generation of $B$-factories. Nowadays the measurement of $\sin \left(2 \phi_{1}\right)$ on the golden channels $B^{0} \rightarrow c \bar{c} K^{0}$ is a precision measurement, with the world average [5] being:

$$
S_{c \bar{c} K^{0}}=0.691 \pm 0.017
$$

Belle II will make sizable progress on these channels: with the full $50 \mathrm{ab}^{-1}$ dataset, the expected statistic (systematic) uncertainty on the $S_{c \bar{c} K^{0}}$ parameter will be 0.0027 (0.0044). Careful study and reduction of all sources of systematic errors will need to be performed, and also penguin pollution effects that have been neglected so far will have to be estimated through measurements of channels related via $S U(3)$ or other symmetries.

Measurements of penguin dominated $B^{0}$ decays sensitive to $\sin \left(2 \phi_{1}\right)$ are important because any significant deviation from the value measured in the golden channels might be a hint of New Physics. Table 1 summarizes the expected uncertainties that Belle II will achieve with a dataset of $5 \mathrm{ab}^{-1}[6]$.

Table 1: Sensitivity estimates on time-dependent $C P$-violation for some of the most important penguin dominated channels. Event yields and uncertainties on the $S$ and $A$ parameters as given, along with the uncertainties on the 2017 world averages.

\begin{tabular}{lcccccc}
\hline \hline Channel & $\int \mathscr{L}$ & Event yield & $\sigma(S)$ & $\sigma(S)_{2017}$ & $\sigma(A)$ & $\sigma(A)_{2017}$ \\
\hline$\phi K^{0}$ & $5 \mathrm{ab}^{-1}$ & 5590 & 0.048 & 0.12 & 0.035 & 0.14 \\
$\eta^{\prime} K^{0}$ & $5 \mathrm{ab}^{-1}$ & 27200 & 0.027 & 0.06 & 0.020 & 0.04 \\
$\omega K_{S}^{0}$ & $5 \mathrm{ab}^{-1}$ & 1670 & 0.08 & 0.21 & 0.06 & 0.14 \\
$K_{S} \pi^{0} \gamma$ & $5 \mathrm{ab}^{-1}$ & 1400 & 0.10 & 0.20 & 0.07 & 0.12 \\
$K_{S} \pi^{0}$ & $5 \mathrm{ab}^{-1}$ & 5699 & 0.09 & 0.17 & 0.06 & 0.10 \\
\hline \hline
\end{tabular}

\subsection{Semileptonic $B$ Decays}

Semileptonic decays are very important because they allow the measurements of the CKM Matrix elements $\left|V_{u b}\right|$ and $\left|V_{c b}\right|$ from tree level amplitudes. Only the clean environment of an $e^{+} e^{-}$ 
collider allows to perform a wide array of inclusive and exclusive measurements that would allow a deeper investigation on the tensions between inclusive and exclusive determinations and within the global fit of the Unitarity Triangle.

A particularly important class of semileptonic decays is the one that involves $\tau$ 's in the final state. While experimentally more challenging, these decays could highlight the existence of particles (as for example charged Higgs-like bosons) which would introduce violations of the Lepton Flavor Universality (LFU). One of the most promising areas to look for New Physics effects is that of the $b \rightarrow c \tau v$ decays. In fact, intriguing hints have been seen by the BaBar, Belle, and LHCb experiments on studying the decays $B \rightarrow D^{(*)} \tau \nu$. Very precise theoretical predictions can be obtained on the quantities:

$$
R\left(D^{(*)}\right)=\frac{\Gamma\left(B \rightarrow D^{(*)} \tau v\right)}{\Gamma\left(B \rightarrow D^{(*)} \ell v\right)}, \ell=e, \mu
$$

(most of the systematics cancel in the ratio). The combination [5] of the measurements available to present shows a tension with the SM predictions at the $4.0 \sigma$ level, so it is imperative to investigate this further.
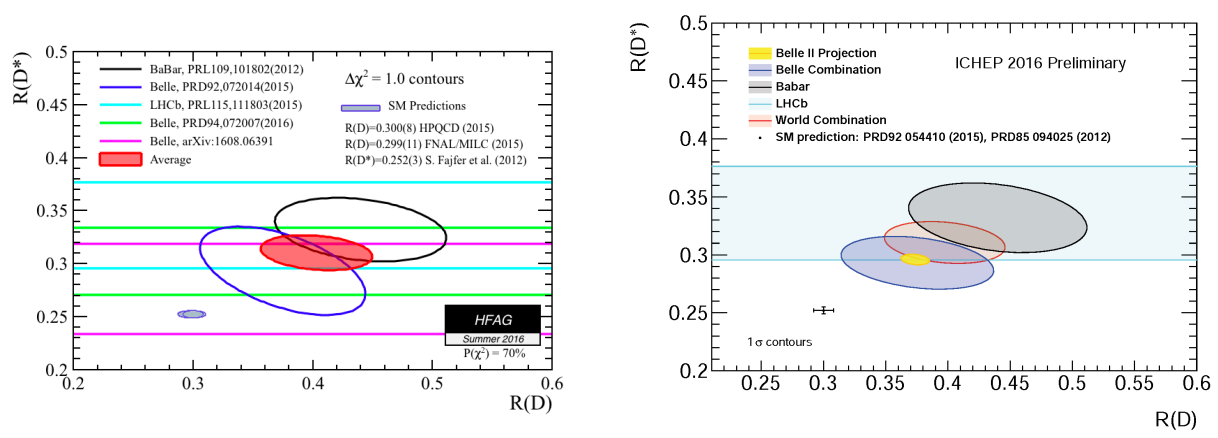

Figure 3: Left figure: current average from [5] of the experimental measurements on $R(D)$ and $R\left(D^{*}\right)$. Right: projection of the Belle II sensitivity with the full dataset on the same quantities.

Thanks to the full knowledge of the kinematics, and the high $B_{\text {tag }}$ reconstruction efficiencies (for both hadronic and semileptonic decays), Belle II will greatly extend the current precision, see Fig. 3. Moreover, the increase in statistics will allow for differential analyses on observables like the $\tau$ and $D^{*}$ polarization, which will help in discriminating among different New Physics models. In this area there will be competition with the LHCb experiment, which can exploit the very precise vertexing capabilities and to whom the semitauonic $\Lambda_{c}$ decays will be accessible.

\subsection{Electroweak Penguins}

Another area where a number of interesting tensions between the Standard Model and the experiments exist is that of the rare $B$ decays that proceed through electroweak penguin amplitudes. In the past few years several tensions between experimental measurements and SM expectations have been observed in $b \rightarrow s \ell^{+} \ell^{-}$transitions in terms of (differential) branching fractions, LFU violation, forward/backward asymmetries, and other angular observables. An extensive review of the current anomalies and an attempt to extract a New Physics contribution that would explain all the observed anomalies can be found in [7]. The authors suggest that the most promising 
explanation for these tensions involves a significant New Physics contribution to the $\mathscr{C}_{9}$ Wilson coefficient.

This is an area where the LHCb experiment has a significant advantage in all the decays that can be fully reconstructed, while Belle II will have a leading role in inclusive measurements and decays with neutrinos or $\tau$ 's in the final states (e.g. $B \rightarrow K^{(*)} v \bar{v}, B \rightarrow K^{(*)} \tau^{+} \tau^{-}, \ldots$ ), and in general all the decays in which systematic uncertainties related to background modeling are important. For example, by measuring differential branching fractions and forward/backward asymmetries in inclusive $B \rightarrow X_{s} \ell^{+} \ell^{-}$, with $50 \mathrm{ab}^{-1}$ Belle II will be able to verify the potential New Physics contribution: as shown in Fig. 4 the discrepancy between the currently preferred values for $\mathscr{C}_{9}^{N P}$ and $\mathscr{C}_{10}^{N P}$ and the $\operatorname{SM}(0,0)$ will be tested with a significance of $\sim 6 \sigma[6]$.
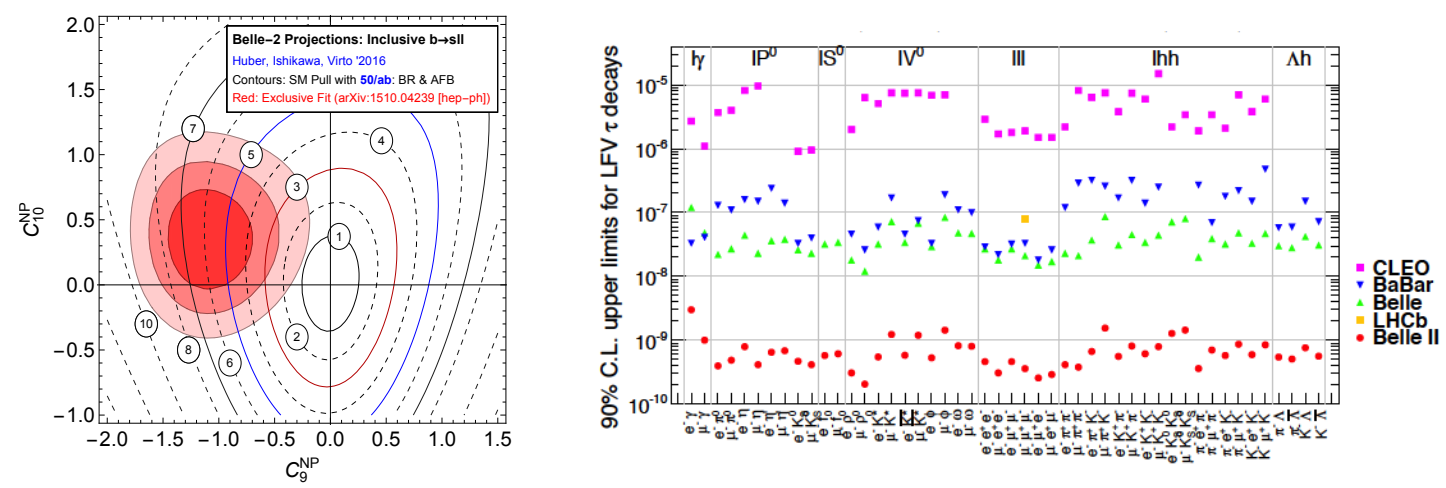

Figure 4: Left figure: $50 \mathrm{ab}^{-1}$ Belle II sensitivity to New Physics Wilson coefficients $\mathscr{C}_{9}^{N P}$ and $\mathscr{C}_{10}^{N P}$ using inclusive $B \rightarrow X_{s} \ell^{+} \ell^{-}$decays (branching fractions and forward/backward asymmetries). Right: sensitivity of Belle II (compared to CLEO, BaBar, Belle, and LHCb) on a number of Lepton Flavor violating $\tau$ decays.

\subsection{Charm Physics}

The BaBar and Belle experiments gave many important contributions to charm Physics, most notably the discovery of the $D^{0}-\bar{D}^{0}$ oscillation, to charm Physics [1]. Currently, and thanks to the high sensitivity of LHCb on final states containing only charged particles, the parameters driving the oscillation are more precisely determined and stringent limits on both direct and indirect $C P$ violation are set. $C P$-violation in the charm sector is predicted to be negligibly small in the $\mathrm{SM}$, so any measured significant asymmetry would be a strong hint of New Physics.

For the $D^{0}-\bar{D}^{0}$ mixing, Belle II will greatly reduce the uncertainties on the mixing parameters and on $C P$-violation, especially in the modes which contain neutrals $\left(\pi^{0}\right.$ 's, $\eta$ 's, ...) in the final state. Thanks to the higher boost of the $D$ mesons in the laboratory frame, Belle II will exploit at its full the improved vertexing capabilities of its vertex detectors (that for $B$-physics are partially spoiled by the reduction of the energy asymmetry of SuperKEKB compared to KEKB), so that the expected proper decay time resolution for $D^{0}$ decays is expected to be $\sim 0.14$ ps.

Also on rare charm decays to neutral particles (for example $D^{0} \rightarrow \gamma \gamma$, whose branching fraction is predicted to be a few $\times 10^{-8}$ in the SM), Belle II will drive the sensitivity improvement, with the caveat that harder beam background conditions might be a limiting factor for some modes. 


\section{$5.5 \tau$ Physics}

One of the areas in which Belle II will dominate the progress in the next few years will be that of rare/forbidden $\tau$ decays. In particular, Lepton Flavor violating decays such as $\tau \rightarrow \mu \gamma$ are practically forbidden in the Standard Model (their branching fractions being $(O) \sim 10^{-50}$, due to the tiny neutrino masses), but could be enhanced to detectable levels by New Physics contributions. Fig. 4 (right) shows the current experimental limits on a broad set of Lepton Flavor violating $\tau$ decays, along with the expected sensitivity of Belle II with a $50 \mathrm{ab}^{-1}$ dataset. Assuming no evidence will show up, the limits on the branching fractions will be pushed below $10^{-9}$ for many of these channels, allowing for non-trivial exclusions on the parameter space of several New Physics models. Given that tight control of the backgrounds will be crucial for these modes, it is anticipated that LHCb will be competitive only in a very few channels (most notably $\tau \rightarrow \mu \mu \mu$ ).

Another stringent null test on the SM predictions can be performed by measuring the Electric Dipole Moment (EDM) of the $\tau$ lepton. Using the optimal observables proposed in [8], Belle has already pioneered the measurement of the EDM of the $\tau$, producing an upper limit still several order of magnitudes above the SM prediction [9]. Belle II will significantly improve over this result, but the final sensitivity will be dominated by the level of knowledge of the detector and the control of systematic uncertainties, as this analysis will be very challenging.

\section{6 $e^{+} e^{-} \rightarrow$ Light Hadrons and Exotic Particles}

A long standing discrepancy (at the $\sim 3 \sigma$ level) between SM predictions and experiments is being observed in the measurement of the anomalous magnetic moment of the muon, commonly referred to as $(g-2)_{\mu}[10]$, [11]. A large fraction of the uncertainty on the theoretical prediction arises from quantum corrections to the $\mu \mu \gamma$ vertex that involve light hadrons (hadronic vacuum polarization). Given the very non-perturbative regime of QCD at this energy, this hadronic contribution cannot be reliably calculated, so input from the experiments is needed.

The measurement of the $e^{+} e^{-} \rightarrow \rho^{0}, \omega, \phi, \ldots$ cross sections can be used, through the Optical Theorem, to infer the size of the hadronic contributions to the $(g-2) \mu$. Thanks to the Initial State Radiation (ISR) and the very hight integrated luminosity, the $B$-factories can measure very precisely the cross sections $e^{+} e^{-} \rightarrow h \gamma$, where $h$ is any hadronic final state with mass between 0.5 and $4 \mathrm{GeV}$. This technique has already been used by BaBar to measure the cross-section of many final states (Belle could not perform the same measurements due to trigger limitations), and will be employed by Belle II to improve the precision of the theoretical determinations of $(g-2)_{\mu}$. Also in this case most of the emphasis of the analysis will be on the control of the systematic uncertainties and on the accurate knowledge of the detector response.

The ISR can be exploited at Belle II also to probe the existence of exotic particles, such as for example the members of the so-called Dark Sector. In this framework (particularly appealing for the explanation of WIMP Dark Matter, see e.g. [12]), New Physics particles are decoupled from the SM particles and (in its minimal formulation) only a mediator particle connects the two sectors. This mediator, commonly termed dark photon $\left(A^{\prime}\right)$, mixes kinetically with coupling strength $\varepsilon$ with the SM hypercharge, and its mass could lie in the interval $[1,10] \mathrm{GeV}$, thus potentially at reach of the $B$-factories. BaBar and Belle searched for the $A^{\prime}$ production (associated to a photon or a light Dark Sector Higgs boson) in $e^{+} e^{-}$collisions in several different final states [1]. Belle II can greatly 
improve the sensitivity for these searches, not only due to the increase in integrated luminosity, but also thanks to its better hermeticity, compared to BaBar. Fig. 5 shows the expected sensitivity of $20 \mathrm{fb}^{-1}$ of Belle II data on the ISR production of $A^{\prime}$, with $A^{\prime}$ decaying to invisible: basically a mono-photon search that is possible only at an $e^{+} e^{-}$collider and requires the development of a dedicated trigger.
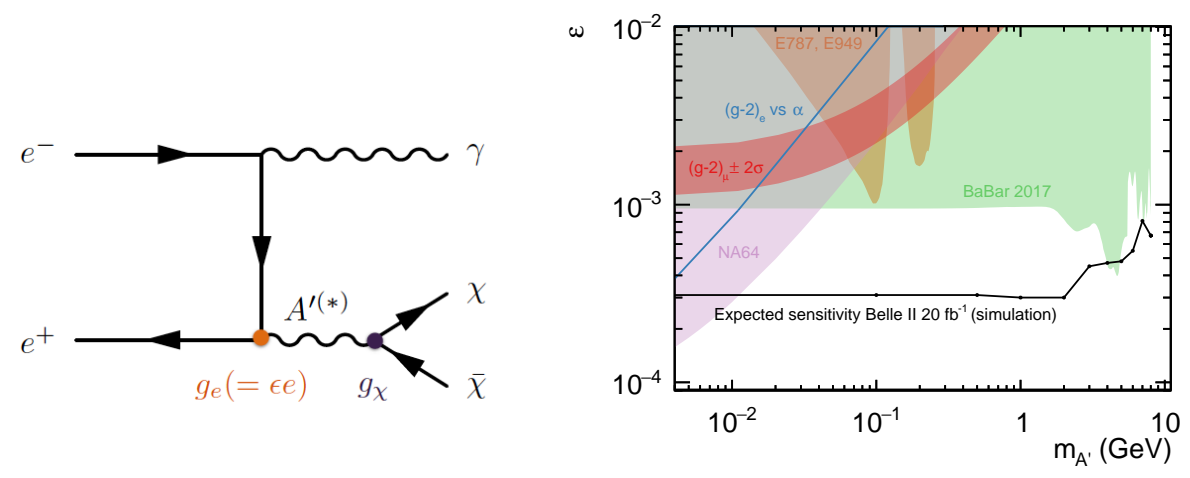

Figure 5: Feynman diagram (left) and sensitivity estimate in the $\varepsilon$ vs $m_{A^{\prime}}$ plane for $20 \mathrm{fb}^{-1}$ of Belle II data with the mono-photon search strategy.

Finally, a very vital area of research was inaugurated by the $B$-factories with the discovery of the $X$ (3872), other exotic particles, and several charmed baryons. For some of the newly discovered particles, the quantum numbers have not been determined yet. With the massive increase in statistics it is very likely that the determination of these quantum numbers will become possible (and also that more states will be discovered).

\section{Commissioning plan}

The Belle II detector is currently in its last phases of construction and it is ready to be rolled into its final position on the beam line. Between May and July 2017 an extensive cosmic data-taking run will be performed, involving all the subdetectors then available: the Central Drift Chamber (CDC), Time Of Propagation (TOP), Electromagnetic Calorimeter (ECL), and the $K_{L}^{0}$ and Muon detector (KLM). The goal of this initial cosmic run will be the integration of the Data Acquisition System, and the initial calibration of the different components.

The Phase II of the commissioning of SuperKEKB will start at the end of year 2017, and first colliding beams at Belle II around February 2018. The main goal of this phase of commissioning will be the study of the beam backgrounds with another specific BEAST II detector installed in place of the silicon vertex trackers. The target instantaneous luminosity will be $10 \times 10^{34} \mathrm{~cm}^{-2} \mathrm{~s}^{-1}$, on the same order of magnitude of the first generation of $B$-factories, and possibly an integrated luminosity around $10 \mathrm{fb}^{-1}$ will be delivered. Besides the commissioning activities on colliding beams, there will be some Physics opportunities with this initial dataset: after an initial phase at the $\Upsilon(4 S)$ energy, the center of mass energy will probably be increased to study the quarkonium spectroscopy around the $\Upsilon(5 S)$ and $\Upsilon(6 S)$ resonances. 
At the end of year 2018, after convincing evidence has been established that the beam backgrounds are low enough for the installation of the pixel (PXD) and strip (SVD) silicon vertex trackers, the Phase III of the SuperKEKB commissioning will start. The instantaneous luminosity will be gradually increased towards the goal of $8 \times 10^{35}$, and the full Physics program of the experiment will begin.

\section{Conclusions}

The Belle II experiment will greatly extend our present knowledge of Flavor Physics, probing with much greater precision several of the tensions between theory and experiments that could point towards New Physics. A lot of the progress will be provided by the unprecedented luminosity of the SuperKEKB collider, but many improvements in the detector technologies and in data analysis technique will be necessary to fully exploit the richness of its dataset. As we enter the final commissioning phases of the detector, we anticipate that the first Physics papers will appear in the year 2018.

Most of the sensitivity studies presented in this contribution have been prepared within the Belle II Theory interface Platform (B2TiP, [6]), an open network that connects theorists and experimentalists to define the best strategies to optimize the Physics reach of the Belle II experiment and the possible synergies with the other experiments. A full report on the Physics reach on many other channels in preparation and will be submitted for publication in the Spring of 2017.

\section{References}

[1] A. J. Bevan et al., Eur. Phys. J. C74, 3026 (2014).

[2] Y. Ohnishi et al., Prog. Theor. Exp. Phys. 03A011 (2013).

[3] M. Bona et al., INFN/AE-07/2, SLAC-R-856, LAL 07-15 (2007).

[4] T. Abe et al., KEK-REPORT-2010-1, arXiv:1011.0352 [physics.ins-det].

[5] Y. Amhis et al., arXiv:1612.07233 [hep-ex].

[6] "Belle II Theory interface Platform", https://confluence.desy.de/display/BI/B2TiP+WebHome. The final report is currently under internal review and is going to be submitted for publication to Prog. Theor. Exp. Phys. in Spring 2017.

[7] S. Decotes-Genon et al., JHEP 1606092 (2016).

[8] D. Atwood and A. Soni, Phys. Rev. D45, 2405 (1992).

[9] K. Inami, presentation at The 13th International Workshop on Tau Lepton Physics (TAU2014), Aachen, Germany, 14-19 September 2014.

[10] F. Jegerlehner and A. Nyffeler, Phys. Reports 477, 1 (2009).

[11] G. W. Bennett et al., Phys. Rev. Lett. 92, 161802 (2004).

[12] M. Pospelov, A. Ritz, M. Voloshin, Phys. Lett. B 662, 53 (2008). 